\title{
MAC-AWAKE OF ISOFLURANE, ENFLURANE AND HALOTHANE EVALUATED BY SLOW AND FAST ALVEOLAR WASHOUT
}

\author{
D. M. GAUMANN, J.-P. MUSTAKI AND E. TASSONYI
}

\begin{abstract}
SUMMARY
End-tidal anaesthetic concentrations at first eye opening in response to a verbal command during recovery from anaesthesia (MAC-awake), were measured for isoflurane $(\mathrm{n}=16)$, enflurane $(\mathrm{n}=$ 16) and halothane $(\mathrm{n}=14)$. MAC-awake was measured during either slow or fast alveolar washout. Slow washout was obtained by decreasing anaesthetic concentrations in predetermined steps of 15 min, assuming equilibration between brain and alveolar partial pressures. Fast alveolar washout was obtained by discontinuation of the inhalation anaesthetic, which had been maintained at 1 MAC for at least $15 \mathrm{~min}$. Mean MAC-awake obtained with slow alveolar washout was similar for isoflurane (0.25 (SD 0.03) MAC), and enflurane (0.27 (0.04) MAC) and significantly greater than values obtained by fast alveolar washout (isoflurane: 0.19 (0.03) MAC; enflurane: 0.20 (0.03) MAC). The MAC-awake of isoflurane and enflurane was significantly less than that of halothane, which was 0.59 (0.10) MAC as evaluated by the slow and 0.50 (0.05) MAC as evaluated by the fast alveolar washout method. Recovery time from anaesthesia with fast alveolar washout was 8.8 (4.0) min for halothane, which was not different from isoflurane (15 (2.5) min), but significantly shorter than for enflurane (22 (10) min), reflecting differences in the anaesthetic concentration gradient between MAC and MAC-awake values. These data do not support the hypothesis of a uniform ratio between $M A C$ and MAC-awake values.
\end{abstract}

\section{KEY WORDS}

Anaesthesia: depth, MAC, MAC-awake. Anaesthetics, volatile enflurane, halothane, isoflurane.

MAC-awake has been defined as the alveolar concentration of a volatile anaesthetic at which eye opening to a verbal command occurred for the first time during awakening from anaesthesia [1]. In this initial study, MAC-awake was measured for methoxyflurane, halothane and fluroxene by two different methods. With a slow alveolar washout method, the inspiratory concentration of the anaesthetic was decreased in predetermined steps and kept constant for approximately $15 \mathrm{~min}$ to achieve equilibration between alveolar and brain partial pressures. With a fast washout method, the alveolar gas concentration was decreased spontaneously by allowing the patient to breathe ambient air. MACawake values, obtained after equilibration of cerebral and alveolar anaesthetic concentrations, were closely comparable between the different inhalation anaesthetics. A value of $0.52 \mathrm{MAC}$ was observed for methoxyflurane and halothane, $0.60 \mathrm{MAC}$ for fluroxene and $0.67 \mathrm{MAC}$ for ether. It was thus hypothesized that MAC-awake was a fixed ratio of MAC that would allow the prediction of MAC-awake values for other inhalation anaesthetics. Smaller MAC-awake values were observed with fast alveolar washout, as anaesthetic equilibration between brain and alveoli was not achieved and partial pressures in brain lagged behind alveolar pressures. The MAC-awake of isoflurane has been measured only recently by a fast alveolar washout method and was found to be $0.15 \mathrm{MAC}$ [2], which is considerably less than observed for halothane with fast alveolar washout (0.33 MAC) [1].

The present study was conducted to clarify the difference between the measured and predicted MAC-awake of isoflurane, by comparing slow and fast alveolar washout. Further, the hypothesis of a uniform MAC-awake value of approximately $0.58 \mathrm{MAC}[1]$, was tested by comparing the MACawake of isoflurane, enflurane and halothane in the presence of brain-alveolar anaesthetic equilibration, as established during slow alveolar washout.

\section{PATIENTS AND METHODS}

After approval by the local institutional Research Ethics Committee and with informed consent, we studied 46 patients of ASA physical status I and II, undergoing elective corrective maxillo-facial and plastic facial surgery. The patients were allocated randomly to three groups according to the inhalation anaesthetic administered: group $1(n=16)$ received isoflurane, group II $(n=16)$ received enflurane and group III $(n=14)$ halothane. During recovery from anaesthesia, $50 \%$ of the patients in each group were subjected to slow alveolar washout of the inhalation anaesthetic and the other $50 \%$ to fast alveolar washout. Patients did not receive any preanaesthetic medication the night before or on the day of surgery. Before induction of anaesthesia, a venous cannula

D. M. Gaumann, M.D.; J.-P. Mustaki, M.D.; E. Tassonyi, M.D. Department of Anaesthesiology, University Hospital of Geneva, 1211 Geneva 4, Switzerland. Accepted for Publication: July 10, 1991.

Correspondence to D.M.G. 
was inserted and routine monitoring commenced consisting of ECG, non-invasive automatic arterial pressure and pulse oximetry. Anaesthesia was induced with propofol $2.5 \mathrm{mg} \mathrm{kg}^{-1}$ i.v. After loss of the eyelid reflex, the patients' lungs were ventilated by face mask with the group-specific inhalation anaesthetic in an air-oxygen mixture. Before tracheal intubation, vecuronium $0.1 \mathrm{mg} \mathrm{kg}^{-1}$ i.v. was given and topical anaesthesia of the larynx and upper trachea was performed with $4 \%$ lignocaine $3 \mathrm{ml}$. The patient's lungs were ventilated mechanically and $\mathbf{E}_{\mathrm{CO}}^{\prime}$, values maintained within the normal range $\left(\mathrm{E}^{\prime} \mathrm{CO}_{2}: 4.5-5.5 \%\right)$. Inspired and end-expiratory concentrations of oxygen, carbon dioxide and isoflurane, enflurane or halothane were measured continuously by a multiple gas analyser (Capnomac, Datex) by aspiration from the end of the tracheal tube $\left(200 \mathrm{ml} \mathrm{min}^{-1}\right)$. Volatile anaesthetic concentrations were detected by infra-red photometry with a rapid measurement rise time of $<770 \mathrm{~ms}$. Calibration was performed before each case with a standard gas mixture (Quick Cal, Datex).

Anaesthesia was maintained with isoflurane, enflurane or halothane in an air-oxygen mixture throughout surgery and no other anaesthetic drugs were given. At the end of surgery and before the start of the alveolar washout period, all patients in group I and group III underwent mechanical ventilation of the lungs for $15 \mathrm{~min}$ with $1 \mathrm{MAC}$ of isoflurane $(1.2 \mathrm{vol} \%)$ or halothane $(0.8 \mathrm{vol} \%)$, respectively, in $100 \%$ oxygen $[3,4]$. Correspondingly, patients in group II, subjected to fast alveolar washout, underwent ventilation for $15 \mathrm{~min}$ with $1 \mathrm{MAC}$ of enflurane $(1.7$ vol \%) [5], while patients for slow alveolar washout received $1.0 \mathrm{vol} \%$ of enflurane before the beginning of the washout period. This initial $15-\mathrm{min}$ equilibration period should have produced equilibration between brain and alveolar partial pressures, at a comparable anaesthetic concentration (1 MAC) before alveolar washout of the different inhalation anaesthetics. Patients for slow alveolar washout of enflurane were treated differently. In this group, we deliberately chose a sub-MAC concentration for the initial equilibration period, in order to keep the wake-up time within acceptable limits, as the concentration gradient between MAC and MACawake is especially great for enflurane (see below). Alveolar washout was conducted in all patients by maintaining normoventilation with $100 \%$ oxygen 10 litre $\mathrm{min}^{-1}$. Slow alveolar washout was performed by decreasing the inspiratory anaesthetic concentration in predetermined steps every $15 \mathrm{~min}$, to achieve equilibration between inspiratory and expiratory concentrations and cerebral partial pressure. These steps were $0.1-0.2$ vol $\%$ for halothane, $0.2-0.3 \mathrm{vol} \%$ for enflurane and $0.2-0.4 \mathrm{vol} \%$ for isoflurane. Fast alveolar washout was conducted by discontinuing the anaesthetic, flushing of the circle breathing system with $100 \%$ oxygen and maintenance of normoventilation with $100 \%$ oxygen.

From the beginning of the alveolar washout period until the end of the study, all patients received a standardized verbal command to open their eyes, played from a tape every $20 \mathrm{~s}$ via occlusive headphones. No other stimulus was used during the entire wake-up period. At first eye opening to command, the end-tidal concentration of the inhalation anaesthetic was recorded as MAC-awake value, and the elapsed time from the start of the alveolar washout was recorded. At this point the study was terminated, and the trachea was extubated.

MAC-awake values were divided by the ageadjusted MAC values. For enflurane, similar factors were assumed as had been established for halothane and isoflurane $[5,6]$ : the 1 MAC value of enflurane $(1.7 \%)$ was multiplied by the factor 1.1 for age $19-30 \mathrm{yr}$ and by the factor of 1.2 for age less than $19 \mathrm{yr}$. Duration of anaesthesia was calculated as the time from induction of anaesthesia until the start of the alveolar washout period. Wake-up time was considered the period from the beginning of the alveolar washout to first eye opening. Comparison for age and duration of anaesthesia was conducted between all groups by one-way analysis of variance (ANOVA). Comparison of wake-up times between groups was performed for fast alveolar washout by one-way ANOVA, followed by the Fisher's PLSD test. Comparison of wake-up times with slow alveolar washout was not performed, as the equilibration steps were chosen arbitrarily. MAC-awake values, expressed as vol \% of the anaesthetic, were compared within each group for slow vs fast alveolar washout by unpaired $t$ test. MAC-awake values expressed as ratio of MAC were compared between groups for slow and fast alveolar washout by one-way ANOVA followed by the Fisher's PLSD test. $P<0.05$ was considered significant in all tests. Statistical analyses were performed on a Macintosh SE computer using the Statview 512 program (Brain Power, Inc.; Calabasas, California 91302).

\section{RESULTS}

There was no difference in patients' age and duration of anaesthesia between the groups. Mean wake-up times with fast alveolar washout were significantly shorter for halothane (8.8 (SD 4.0) min) than for enflurane (22 (10) $\mathrm{min}$ ), while wake-up times for isoflurane $(15(2.5) \mathrm{min}$ ) did not differ from those for halothane or enflurane (table I). For each of the inhalation anaesthetics, MAC-awake values obtained by slow alveolar washout were significantly greater than those obtained by fast alveolar washout. Using slow alveolar washout, end-tidal anaesthetic concentrations at eye opening generally corresponded to inspiratory concentrations, indicating brain-alveolar equilibration. However, two patients anaesthetized with isoflurane and three patients each, anaesthetized with enflurane or halothane, had 0.1 vol $\%$ lesser inspiratory than end-tidal anaesthetic concentrations at eye opening, indicating that equilibration was not achieved completely (table I).

There was no difference between the MAC-awake values of isoflurane and enflurane, when MACawake was expressed as ratio of MAC. With slow alveolar washout the MAC-awake of isoflurane was $0.25(0.03) \mathrm{MAC}$ and that of enflurane $0.27(0.04)$ MAC; with fast alveolar washout, values were 0.19 (0.03) MAC and $0.20(0.03) \mathrm{MAC}$, respectively. The MAC-awake of halothane was significantly greater 
TABle I. Patient characteristics and study data (mean (SD) [range]). Comparison was conducted for age and duration of anaesthesia between all groups, for wake-up times between groups with fast alveolar washout, for $M A C$-awake (\%) between slow and fast alveolar washout within each anaesthetic group, and for $M A C$-aroake $(\%) \times M A C^{-1}(\%)$ between different anaesthetic groups with slow or fast alveolar washout. $\mathrm{P}<0.05$ : * between slow and fast alveolar washout within each anaesthettc group; †between anaesthetic groups for fast or slow alveolar washout; $\ddagger$ halothane different from isoflurane and enflurane; Shalothane different from enflurane

\begin{tabular}{|c|c|c|c|c|c|c|}
\hline & \multicolumn{2}{|c|}{ Isoflurane washout } & \multicolumn{2}{|c|}{ Enflurane washout } & \multicolumn{2}{|c|}{ Halothane washout } \\
\hline & Slow & Fast & Slow & Fast & Slow & Fast \\
\hline No. of patients & 8 & 8 & 8 & 8 & 7 & 7 \\
\hline \multirow{2}{*}{ Age (yr) } & 32 & 32 & 23 & 25 & 26 & 28 \\
\hline & {$[18-49]$} & {$[20-52]$} & {$[17-28]$} & {$[20-33]$} & {$[16-54]$} & {$[18-33]$} \\
\hline \multirow{2}{*}{$\begin{array}{l}\text { Duration of } \\
\text { anaesthesia (min) }\end{array}$} & $101(33)$ & $101(23)$ & $98(29)$ & $88(42)$ & $101(49)$ & $90(43)$ \\
\hline & {$[60-150]$} & {$[60-120]$} & {$[50-135]$} & {$[45-140]$} & {$[56-170]$} & {$[45-145]$} \\
\hline \multirow{4}{*}{$\begin{array}{l}\text { Wake-up time } \\
\text { (min) } \\
\text { MAC-awake (\%) }\end{array}$} & $46(13)$ & $15(2.5)$ & $32(10)$ & $22(10)$ & $25(9.0)$ & $8.8(4.0)+5$ \\
\hline & {$[25-60]$} & [12-20] & [17-50] & {$[13-45]$} & {$[16-40]$} & {$[3-15]$} \\
\hline & $0.31(0.04)^{\star}$ & $0.23(0.05)$ & $051(0.08)^{\star}$ & $0.36(0.05)$ & $0.50(0.06)^{\star}$ & $0.41(0.04)$ \\
\hline & {$[0.3-0.4]$} & {$[0.2-0.3]$} & {$[0.4-0.6]$} & {$[0.3-0.4]$} & {$[0.4-0.6]$} & {$[0.4-0.5]$} \\
\hline \multirow{2}{*}{$\begin{array}{l}\text { MAC-awake (\%) } \\
\operatorname{MAC}^{-1}(\%)\end{array}$} & $0.25(0.03)$ & $0.19(0.03)$ & $0.27(0.04)$ & $0.20(0.03)$ & $0.59(010)+\ddagger$ & $0.50(0.05) \dagger \ddagger$ \\
\hline & {$[0.23-0.31]$} & {$[0.16-0.23]$} & {$[0.21-0.32]$} & {$[0.16-0.24]$} & {$[0.43-0.71]$} & {$[10.43-0.60]$} \\
\hline
\end{tabular}

compared with isoflurane and enflurane, with a value of 0.59 (0.10) MAC obtained by slow alveolar washout and $0.50(0.05) \mathrm{MAC}$ by fast alveolar washout.

\section{DISCUSSION}

The present study has shown clearly that there was a difference between MAC-awake values of isoflurane and enflurane compared with halothane. Therefore, the hypothesis of a uniform MAC-awake value, representing a fixed ratio of MAC [1], cannot be supported.

The slow alveolar washout method we used corresponded to that described initially by Stoelting, Longnecker and Eger [1]; this assumed brainalveolar equilibration of the anaesthetic after maintaining alveolar concentrations constant for $15 \mathrm{~min}$. As indicated above, this was achieved in the majority of our patients. However, because of the predetermined steps of decreased inspiratory anaesthetic concentrations, some inaccuracy in determining MAC-awake may have occurred in some patients, as a result of incomplete brain-alveolar equilibration. However, we believe that this error was small, as differences in inspiratory to end-tidal anaesthetic concentrations at eye opening, which occurred in some patients, did not exceed 0.1 vol \%. Furthermore, based on the degree of decreased inspiratory concentrations, isoflurane should have exhibited the greatest variability. However, it had the smallest variability $-0.1 \mathrm{vol} \%$, compared with halothane $(0.2 \mathrm{vol} \%)$ and enflurane $(0.2 \mathrm{vol} \%)$.

The MAC-awake of halothane $(0.59 \mathrm{MAC})$ that we obtained by slow alveolar washout was slightly greater, as was found by Stoelting, Longnecker and Eger (0.52 MAC) [1]. However, MAC-awake of halothane, obtained by fast alveolar washout in the present study (0.50 MAC) was markedly greater compared with that obtained by Stoelting's group (0.33 MAC) [1]. This difference may be a result of differences in study design. Stoelting, Longnecker and Eger examined end-tidal anaesthetic concentrations intermittently, approximately every 3-5 min, and defined the MAC-awake value as the anaesthetic concentration midway between the value permitting eye opening to a non-standardized verbal command and the value just preventing this response. In our study, more accuracy was provided by continuous measurement of inspiratory and endexpiratory anaesthetic concentrations, and standardization of the verbal command. Patients in Stoelting's study did not receive any i.v. induction agent, while our patients received propofol i.v. and topical laryngeal anaesthesia on induction. These drugs may have decreased the MAC-awake value, by providing some postoperative sedation and by attenuating the cough reflex, induced by the tracheal tube. However, as the MAC-awake values of halothane were greater in our study than in that of Stoelting, Longnecker and Eger, these effects may be of minor importance. Pain on recovery from anaesthesia may modify awakening by causing additional stimulation and lead to an increase in the measured MAC-awake value. Although this aspect was not studied specifically, most of our patients experienced pain during the early postoperative period, necessitating administration of opioids or anti-inflammatory drugs. This may be an important difference from the study by Stoelting's group, in which patients were reported to be pain free after surgery, and may partially explain the greater MAC-awake values we observed. The great difference between the two studies with regard to MAC-awake obtained by fast alveolar washout may result from differences in several factors which modify anaesthetic elimination and equilibration of brain and alveolar partial pressures, such as duration of anaesthesia, anaesthetic gas concentration, anaesthetic concentration at which alveolar washout was started, mode of ventilation and patient's body weight [6-8].

MAC-awake of isoflurane and enflurane, obtained by the slow washout method (0.25 MAC and $0.27 \mathrm{MAC}$ ), were comparable, but significantly less than the MAC-awake of halothane. The MACawake of isoflurane corresponded to values obtained in volunteers in whom loss of consciousness to subanaesthetic doses of isoflurane was measured [9]. Eye opening to command was lost in one of eight subjects at 0.2 MAC and in all eight subjects at 0.4 MAC isoflurane [9]. Our data thus demonstrate 
clinically important differences in hypnotic potencies of volatile anaesthetics, with isoflurane and enflurane being approximately twice as potent as halothane, ether, methoxyflurane or fluroxene [1]. It was shown also that subanaesthetic doses of isoflurane produced more mental and physical sedation than equianaesthetic doses of nitrous oxide [10]. These data therefore permit selection of volatile anaesthetics for the desired degree of sedation during anaesthesia or in the intensive care unit [11]. Furthermore, differences in hypnotic potencies of volatile anaesthetics are important in view of the fact that, even under surgical anaesthesia, there is some subconscious processing of information, which may modify postoperative psychological behaviour [12]. The MACawake of isoflurane $(0.19 \mathrm{MAC})$ and enflurane (0.20 MAC), obtained by fast alveolar washout, were comparable. For isoflurane, the value was slightly greater than that reported by Gross and Alexander (0.15 MAC) [2], which is probably a result of differences in the study design. In our study, normoventilation was used, while in Gross and Alexander's study fast alveolar washout was conducted with hyperventilation. Hyperventilation may have increased the brain to alveolar isoflurane partial pressure gradient as a result of cerebral vasoconstriction, and thus decreased the MAC-awake value. Further, thiopentone, used as an induction agent by Gross and Alexander, has more pronounced sedative effects during recovery from anaesthesia than propofol, which was used in the present study [13].

Wake-up times of the different volatile anaesthetics obtained in the present study depended on the number of equilibration steps in the case of slow alveolar washout. In the case of fast alveolar washout, wake-up times mainly reflected differences in the concentration of the anaesthetics at the beginning of the washout period (which corresponded to $1 \mathrm{MAC}$ ) and in MAC-awake values. Thus shortest wake-up times $(8.8 \mathrm{~min})$ were observed with halothane, as the concentration gradient for halothane between MAC and MAC-awake was small ( $0.4 \mathrm{vol} \%)$. Longer wake-up times occurred with enflurane ( $22 \mathrm{~min}$ ), reflecting a greater anaesthetic concentration gradient between MAC and MAC-awake ( $1.3 \mathrm{vol} \%$ ). Wake-up times for isoflurane $(15 \mathrm{~min})$ were intermediate between those of halothane and enflurane and corresponded to a MAC to MAC-awake concentration gradient of $0.9 \mathrm{vol} \%$. The low MAC- awake value of isoflurane explains the longer wakeup times after isoflurane compared with halothane anaesthesia, which were studied previously in children and were in apparent contrast with predictions based on the physical properties of these anaesthetics [14].

\section{REFERENCES}

1. Stoelting RK, Longnecker DE, Eger EI II. Minimum alveolar concentrations in man on awakening from methoxyflurane, halothane, ether and fluroxene anesthesia: MAC awake. Anesthesiology 1970; 33: 5-9.

2. Gross JB, Alexander CM. Awakening concentrations of isoflurane are not affected by analgesic doses of morphine. Anesthesia and Analgesia 1988; 67:27-30.

3. Stevens WC, Dolan WM, Gibbons RT, White A, Eger EI II, Miller RD, De Jong RH, Elashoff RM. Minimum alveolar concentrations (MAC) of isoflurane with and without nitrous oxide in patients of various ages. Anesthestology $1975 ; 42$ : 197-200.

4. Gregory GA, Eger EI II, Munson ES. The relationship between age and halothane requirement in man. Anesthestology $1969 ; 30: 488-491$.

5. Gion H, Saidman LJ. The minimum alveolar concentration of enflurane in man. Anesthesiology $1971 ; 35: 361-364$.

6. Stoelting RK, Eger EI II. The effects of ventilation and anesthetic solubility on recovery from anesthesia: an in vivo and analog analysis before and after equilibrium. Anesthestology $1969 ; 30: 290-296$.

7. Carpenter RL, Eger EI Il. Alveolar- to arterial- to venous anesthetic partial pressure difference in humans. Anesthestology $1989 ; 70: 630-635$.

8. Frei FJ, Zbinden AM, Thomson DA, Rieder HU. Is the endtidal partial pressure of isoflurane a good predictor of the partial pressure? British foumal of Anaesthesia 1991; 66: 331-339.

9. Newton DEF, Thornton C, Konieczko K, Frith CD, Doré CJ, Webster NR, Luff NP. Levels of consciousness in volunteers breathing sub-MAC concentrations of isoflurane. British fournal of Anaesthesia 1990; 65: 609-615.

10. McMenemin IM, Parbrook GD. Comparison of the effects of subanaesthetic concentrations of isoflurane or nitrous oxide in volunteers. British fournal of Anaesthesia $1988 ; 60: 56-63$.

11. Kong KL, Willatts SM, Prys-Roberts C. Isoflurane compared with midazolam for sedation in the intensive care unit. Bntish Medical Journal 1989; 298 : 1277-1280.

12. Griffiths $D$, Jones JG. Awareness and memory in anaesthetized patients. British foumal of Anaestheria 1990; 65: 603-605.

13. Mackenzie N, Grant IS. Comparison of the new emulsion formulation of propofol with methohexitone and thiopentone for induction of anaesthesia in day cases. British fournal of Anaesthesia 1985; 57 : 725-731.

14. McAteer PM, Carter JA, Cooper GM, Prys-Roberts C. Comparison of isoflurane and halothane in outpatient paediatric dental anaesthesia. British Fournal of Anaesthesia 1986; 58: $390-393$. 